\title{
Exosome derived from epigallocatechin gallate treated breast cancer cells suppresses tumor growth by inhibiting tumor-associated macrophage infiltration and $\mathrm{M} 2$ polarization
}

\author{
Ji-Young Jang ${ }^{1}$, Jong-Kuen Lee ${ }^{1}$, Yoon-Kyung Jeon ${ }^{1,2}$ and Chul-Woo Kim ${ }^{1,2^{*}}$
}

\begin{abstract}
Background: Tumor-associated macrophages (TAM) play an important role in tumor microenvironment. Particularly, M2 macrophages contribute to tumor progression, depending on the expression of NF-KB. Tumor-derived exosomes can modulate tumor microenvironment by transferring miRNAs to immune cells. Epigallocatechin gallate (EGCG) has well known anti-tumor effects; however, no data are available on the influence of EGCG on communication with cancer cells and TAM.

Methods: Murine breast cancer cell lines, 4T1, was used for in vivo and ex vivo studies. Exosome was extracted from EGCG-treated 4T1 cells, and the change of miRNAs was screened using microarray. Tumor cells or TAM isolated from murine tumor graft were incubated with exosomes derived from EGCG-treated and/or miR-16 inhibitor-transfected 4T1 cells. Chemokines for monocytes (CSF-1 and CCL-2), cytokines both with high (IL-6 and TGF- $\beta$ ) and low (TNF-a) expression in M2 macrophages, and molecules in NF-KB pathway (IKKa and IK-B) were evaluated by RT-qPCR or western blot.

Results: EGCG suppressed tumor growth in murine breast cancer model, which was associated with decreased TAM and M2 macrophage infiltration. Expression of chemokine for monocytes (CSF-1 and CCL-2) were low in tumor cells from EGCG-treated mice, and cytokines of TAM was skewed from M2-into M1-like phenotype by EGCG as evidenced by decreased IL- 6 and TGF- $\beta$ and increased TNF- $\alpha$. Ex vivo incubation of isolated tumor cells with EGCG inhibited the CSF-1 and CCL-2 expression. Ex vivo incubation of TAM with exosomes from EGCG-treated 4T1 cells led to IKKa suppression and concomitant I-KB accumulation; increase of IL-6 and TGF- $\beta$; and, decrease of TNF-a. EGCG up-regulated miR-16 in 4T1 cells and in the exosomes. Treatment of tumor cells or TAM with exosomes derived from EGCG-treated and miR-16-knock-downed 4T1 cells restored the above effects on chemokines, cytokines, and NF-KB pathway elicited by EGCG-treated exosomes.

Conclusions: Our data demonstrate that EGCG up-regulates miR-16 in tumor cells, which can be transferred to TAM via exosomes and inhibits TAM infiltration and M2 polarization. We suggest a novel mechanism by which EGCG exerts anti-tumor activity via regulation of TAM in tumor microenvironment.
\end{abstract}

Keywords: EGCG, Exosomes, miR-16, Tumor microenvironment, Tumor-associated macrophages (TAM)

\footnotetext{
* Correspondence: cwkim@snu.ac.kr

${ }^{1}$ Tumor Immunity Medical Research Center, Cancer Research Institute, Seoul

National University College of Medicine, 28 Yongon-dong, Jongno-gu, Seoul 110-799, Korea

${ }^{2}$ Department of Pathology, Seoul National University College of Medicine, 28 Yongon-dong, Jongno-gu, Seoul 110-799, Korea
} 


\section{Background}

Worldwide, breast cancer is the most frequently diagnosed life-threatening cancer and the most important cause of cancer-related deaths in women [1]. Populationbased investigations have suggested dietary factors which affect the risk of breast cancer [2,3]. Epidemiological studies of Asian and Chinese women have reported an inverse association between the consumption of green tea and the risk of breast cancer. Of the polyphenols present in green tea, epigallocatechin-3-gallate (EGCG) has been identified as having inhibitory effects on tumorigenesis in studies using in vitro and in vivo models of carcinogenesis [4-7]. Anti-tumorigenic activities of EGCG include inhibition of cell proliferation, induction of apoptosis and cell cycle arrest, inhibition of invasion and metastasis, and suppression of angiogenesis [8-13].

Exosomes are circular fragments of membrane released from the endosomal compartment, and are shed from the surface membranes of most cell types [14,15]. An increasing body of evidence indicates that exosomes play a pivotal role in cell-to-cell communication [16], and in particular, tumor cells are found to release large quantities of exosomes [17-19]. The amount of circulating exosomes is greater in the serum or plasma of patients with cancer and predict a poor prognosis [17]. Release of exosomes may protect tumor cells from apoptosis by selective extrusion of apoptosis-inducing proteins. Additionally, exosomes may help tumor cells escape the immune surveillance [18] and carry out pro-angiogenic signals that increase tumor vascularization [20]. In addition, exosomes may transfer genetic information, such as microRNAs (miRNAs) from tumor cells to neighboring cells [21].

Macrophages populate the microenvironment of most tumors. In certain cases, these cells can represent more than half of the tumor mass and play an important role in tumor immunity, which is particularly true for breast cancer [22]. Clinical studies have sought to correlate macrophage density and cancer prognosis. A metaanalysis have shown that, in $80 \%$ of the cases, increased macrophage density was associated with poor prognosis, and that, in the remaining $20 \%$, there was a split between null prognostic value and good prognostic value [23]. Studies of this nature have been performed most extensively for breast cancer, and multiple independent investigations have found increased quantities of tumorassociated macrophages (TAMs) to be associated with poor prognosis [24]. In addition to the extent of macrophage infiltration, the phenotype of TAMs has been shown to affect tumor progression [25]. Within the tumor microenvironment, several stimuli are known to influence the TAM phenotype. Macrophages can be induced to either tumor-suppressive immunological type (referred to as M1) or tumor-promoting inflammatory/ immune-suppressive population (M2 macrophages)
$[26,27]$. Tumor cells produce colony-stimulating factor1 (CSF-1) and Chemokine (C-C motif) ligand 2 (CCL2), which are two major attractants and growth factors for TAM. The concept that TAM are mainly M2 activated, or even M2 'polarized', has been around for almost a decade, and is corroborated by the pattern of TAM marker expression. High production of IL-10 and low production of IL-12 is seen as a hallmark of all non-M1 macrophages, and is also applicable to most TAM populations in different cancer types. Accordingly, high frequency of infiltrating TAM is associated with a poor prognosis for many types of tumors. This pathological association to clinical progression has reemerged in the post-genomic era: genes associated to macrophage infiltration are the same molecular signatures that herald poor prognosis in lymphomas and breast carcinoma patients [28].

We hypothesized that EGCG might regulate the expression of tumor-derived exosomal miRNAs and affect the tumor microenvironment and TAMs. The aim of this study was to investigate the effect that EGCG has on tumor-derived exosomal miRNAs and TAM.

\section{Methods}

\section{Cell lines and reagents}

The mouse mammary tumor cell line, 4T1, were maintained as monolayer cultures in Dulbecco's Modified Eagle Medium (DMEM), supplemented with 10\% fetal bovine serum (FBS), 100 units $/ \mathrm{ml}$ penicillin, and $100 \mu \mathrm{g} / \mathrm{ml}$ streptomycin (Gibco, Grand Island, NY, USA) in a humidified $5 \% \mathrm{CO}_{2} / 95 \%$ air atmosphere at $37^{\circ} \mathrm{C}$. The murine RAW264.7 macrophage cell line were grown in RPMI 1640 containing $10 \%$ fetal bovine serum 100 units $/ \mathrm{ml}$ penicillin, and $100 \mu \mathrm{g} / \mathrm{ml}$ streptomycin (Gibco, Grand Island, NY, USA) in a humidified $5 \% \mathrm{CO}_{2} / 95 \%$ air atmosphere at $37^{\circ} \mathrm{C}$. Epigallocatechin gallate (EGCG) and lipopolysaccharides (LPS) were purchased from Sigma (St. Louis, MO, USA).

\section{Transfection}

The mouse mammary tumor cell line, $4 \mathrm{~T} 1$ or macrophages cell line, RAW264.7 were plated on six-well plates $\left(2 \times 10^{5}\right.$ cells per well $)$ and were allowed to adhere for 24 hours. These cells were transfected with either scramble miRNA inhibitor or miR-16 inhibitor using Lipofectamine 2000 (Invitrogen, Carlsbad, CA). Transfected cells were then cultured for 6 hours, and culture media were replaced with fresh media supplemented with $10 \%$ FBS. The cells were harvested at 24-48 hours after transfection. The scramble miRNA inhibitor or miR-16 inhibitor (100 nM) were obtained from Shanghai GenePharma Co (Shanghai, China). The scramble miRNA mimics or miR-16 mimics (100 $\mathrm{nM})$ were obtained from Genolution (Seoul, Korea). 


\section{Exosomes isolation and purification}

The 4T1 mouse mammary tumor cells (or TAM cells) were centrifuged overnight at 100,000 g to isolate bovinederived exosomes which are present in the DMEM. The exosomes from 4T1 cells were isolated from the remaining supernatants using ExoQuick (System Bioscience, Mountain View, CA, USA) according to the manufacturer's protocol. The pellets were washed in large volumes of PBS and resuspended in $80 \mu \mathrm{l}$ PBS. Proteins in pellets and lysates were quantified by Micro-BCA (Thermo Scientific) in the presence of $2 \%$ SDS. Purity of isolated exosome was assured using electron microscopy by exosomal size or immunobloting for CD63, tsg101, and calnexin.

\section{Quantification of miR-16 by RT-qPCR}

The 4T1 mammary tumor cells were grown in $6 \mathrm{~cm}$ Petri dishes to $70 \%$ confluence then were treated for 24 hr with $100 \mu \mathrm{M}$ EGCG. Total RNA was extracted from these cells using Trizol reagent (Invitrogen). The RNA quality, yield, and size of extracted RNA were analyzed using capillary electrophoresis (Agilent 2100 Bioanalyzer, Agilent Technologies, Foster City, CA, USA). RT-qPCR analysis for miRNAs was carried out with Mir- $\mathrm{X}^{\mathrm{m}}$ miRNA First-Strand Synthesis Kit (Clontech, CA, USA) and SYBR Green Real time PCR Master Mix (TaKaRa, Otsu, Japan) according to the respective manufacturer's instruction. The house-keeping (HK) gene U6 was used as a control for standardization of the initial miRNA content of a sample. Relative changes of gene expression were calculated by the following formula, and the data was represented as fold up-regulation/down-regulation, fold change $=2^{-\Delta \Delta \mathrm{Ct}}$, where $\Delta \Delta \mathrm{Ct}=(\mathrm{Ct}$ of gene of interest, treated -Ct of $\mathrm{HK}$ gene, treated)-(Ct of gene of interest, control-Ct of $\mathrm{HK}$ gene, control). The primers used were as follows: for miR-16, forward $5^{\prime}$-TAGCA GCACGTAAATATTGGCG-3'; for U6, forward 5'-TG GCCCCTGCGCAAGGATG-3, and miR-16 and U6 reverse primer was included in $M i r-X^{\mathrm{TM}}$ miRNA FirstStrand Synthesis Kit.

\section{Prediction of miRNA targets}

Web resources was used to predict miR-16 targets, including a viewer for browsing potential target sites, conserved with or without positional constraints, on aligned UTRs, with periodic updates (http://www.microrna.org).

\section{Quantitative real-time RT-PCR (RT-qPCR)}

Total RNA was extracted using Trizol (Invitrogen). For RT-qPCR analysis, $5 \mu \mathrm{g}$ of RNA was reverse-transcribed using RT-PCR kits (Promega, Madison, WI, USA). PCR was performed using SYBR Green Real time PCR Master Mix (TaKaRa, Otsu, Japan). Relative changes of gene expression were calculated by the following formula:
$\Delta \Delta \mathrm{Ct}=(\mathrm{Ct}$ of gene of interest, treated - $\mathrm{Ct}$ of HK gene, treated)-(Ct of gene of interest, control-Ct of $\mathrm{HK}$ gene, control); where, $\mathrm{Ct}$ was the threshold cycle number, and HK was the house keeping gene.

This data was represented as fold up-regulation/downregulation, fold change $=2^{-\Delta \Delta C t}$. The primer sequences were used as follows: for TGF- $\beta$, forward sequence 5' - CACCGGAGAGCCCTGGATA-3' reverse sequence 5' - TGTACAGCTGCCGCACACA-3'; IL-6 forward sequence 5' -GACAACTTTGGCATTGTGG-3' reverse sequence 5'-ATGCAGGGATGATGTTCTG-3'; TNF- $\alpha$ forward sequence $5^{\prime}$-TCCCAGGTTCTCTTCAAGGG A-3' reverse sequence 5'-GGTGAGGAGCACGTAGT CGG-3'; CSF-1 forward sequence 5'-CGACATGGCT GGGCTCCC-3' reverse sequence 5' - CGCATGGTC TCATCTATTAT-3'; CCL-2 forward sequence 5'-GT TGGCTCAGCCAGATGCA-3' reverse sequence 5'-A GCCTACTCATTGGGATCATCTTG-3'; IL-1 $\beta$, forward sequence 5'-AAGGAGAACCAAGCAACGACA AAA-3' reverse sequence $5^{\prime}$-TGGGGAACTCTGCAG ACTCAAACT-3'.; GAPDH forward sequence 5' - GGG CTGGCATTGCTCTCA-3' reverse sequence 5' - TGC TGTAGCCGTATTCATTGT-3'.

\section{Western blotting}

For western blot analyses, the cells were harvested after 24 hours of exosomes treatment and lysed with lysis buffer $(5 \mathrm{mM} / \mathrm{L}$ ethylenediamine tetra acetic acid; 300 $\mathrm{mM} / \mathrm{L} \mathrm{NaCl} ; 0.1 \% \mathrm{NP}-40 ; 0.5 \mathrm{mM} / \mathrm{L} \mathrm{NaF} ; 0.5 \mathrm{mM} / \mathrm{L}$ Na3VO4; $0.5 \mathrm{mM} / \mathrm{L}$ phenylmethylsulfonyl fluoride; and $10 \mu \mathrm{g} / \mathrm{ml}$ each of aprotinin, pepstatin, and leupeptin; Sigma, St Louis, MO). Following centrifugation at 15,000 g for 30 minutes, the concentrations of supernatant proteins were analyzed using the Bradford reagent (BioRad, Hercules, CA). For analysis of protein contents, $50 \mu \mathrm{g}$ of total proteins was electrophoresed in $10 \%$ SDS-PAGE gel, transferred to polyvinylidene difluoride membranes (Millipore, Bedford, MA), and were incubated with antibodies against IKK $\alpha($ Abcam), I-kB (Santa Cruz Biotechnology), IL-6 (Invitrogen : AMC0864), TNF- $\alpha$ (Abcam : ab1793), TGF- $\beta$ (Abcam : ab64715) or actin (Sigma). Immunoblots were visualized using an enhanced chemiluminescence detection system (Amersham Pharmacia Biotech, Uppsala, Sweden).

\section{Tumor-associated macrophages isolation from tumor tissue}

For ex vivo assay, the $4 \mathrm{~T} 1$ cells $\left(1 \times 10^{5}\right)$ were suspended in $100 \mu \mathrm{l} \mathrm{PBS}$ and then injected subcutaneously into either side of the posterior flank of six BALB/c mice to induce tumor growth. When these cells have established as tumors after 3-to-4 weeks, the resulting tumor mass were harvested for isolation of TAMs. Each tumor tissue was cut into $2 \mathrm{~mm}$ fragments, followed by collagenase 
digestion $(0.3 \mathrm{mg} / \mathrm{ml}$, Worthington Biochemical Corp, NJ, USA) for $1 \mathrm{~h}$ at $37^{\circ} \mathrm{C}$. The suspension was filtered through a $70-\mu \mathrm{m}$ stainless steel wire mesh to generate a single-cell suspension. This resulting suspension was centrifuged and washed twice with PBS ( $\mathrm{pH}=7.4)$. CD11b myeloid cells were purified from tumor cell suspension using the MACS method (Militenyi Biotec). Briefly, the CD11b cells were incubated with beads conjugated with anti-mouse CD11b and were positively selected on LS columns. The purity of recovered cells assessed by flow cytometry was greater than $95 \%$. The viability of isolated cells routinely exceeded $90 \%$, as determined by trypan blue exclusion assays. These TAMs $\left(5 \times 10^{5} / \mathrm{ml}\right)$ were stimulated with the exosomes $(50 \mu \mathrm{g} / \mathrm{ml})$ from the part of the experiment describe above.

\section{In vivo study}

For in vivo assay, the $4 \mathrm{~T} 1$ cells $\left(1 \times 10^{5}\right)$ were suspended in $100 \mu \mathrm{l} \mathrm{PBS}$ and then injected subcutaneously into either side of the posterior flank of six BALB/c mice. Tumor growth was examined daily, and the tumor volumes were calculated every week using the formula for hemi-ellipsoids: $V=$ length $(\mathrm{cm}) \times$ width $(\mathrm{cm}) \times$ height $(\mathrm{cm}) \times 0.5236$. After 5 weeks, each mouse was sacrificed, and the tumors were dissected and weighed. Animals experiment for this research was designed and carried out according to the standard guideline of Institutional Animal Care and Use Committee (IACUC), and the study design had been approved by Seoul National University Institutional Animal Care and Use Committee (http:// iacuc.snu.ac.kr).

\section{Immunohistochemistry}

Tumor tissue was fixed in $4 \%$ buffered neutralized formalin for 48 hours. After embedding in paraffin, $4 \mu \mathrm{m}$ serial sections were made and mounted on a slide. Phenotypic characterization of macrophages was performed using double immunohistochemical staining to enable evaluation of tumor cells. Antigens were retrieved by boiling tissue sections in citrate buffer (DakoCytomation, Glostrup, Denmark) for 10 minutes. Anti-mouse macrophage CD68 mAb (ab31630; Abcam, Cambridge, UK) was used as a marker for all macrophages, and anti-mouse CD163 mAb (NBP1-95135; Novus, Missouri, USA) as a marker for M2-type macrophages.

\section{Statistical analysis}

We employed GraphPad Prism software to conduct statistical analysis. Results are expressed as the mean \pm s.e. $\mathrm{m}$. For p-value calculation, unpaired Student's $t$ tests (two tailed) were utilized for all comparisons. Null hypotheses of no difference were rejected if $\mathrm{p}$-values were less than 0.05 .

\section{Results}

EGCG suppresses tumor growth, macrophages infiltration, and M2 polarization in in vivo murine breast cancer model

To determine if the presence of EGCG has influenced tumor growth, TAM infiltration and differentiation, we established a murine tumor model by injecting murine breast cancer cell lines, 4T1, subcutaneously into syngeneic mice. Mice were then treated with EGCG either PBS as a control by intraperitoneal injection as described in Figure 1A. At 30 days after tumor challenge, a significant decrease of tumor volume and weight was observed in the EGCG-treated group versus the control group $(\mathrm{p}<0.0005)$ (Figure $1 \mathrm{~A}$ and $1 \mathrm{~B})$. Intra-tumoral infiltrations of TMA and M2 macrophages as assessed by immunohistochemical staining were lower in EGCGtreated group than control (Figure 1C).

We next measured the levels of CSF-1 and CCL-2, major chemokines involved in the monocyte recruitment into tumor microenvironment [29], in tumor cells isolated from mice by RT-qPCR. In line with the decreased number of TAM in EGCG-treated mice, expressions of both CSF-1 and CCL-2 were significantly decreased upon EGCG treatment (Figure 1D). Differentiation of TAM into pro-tumoral M2 macrophages is induced by a set of cytokines including IL- 6 and TGF- $\beta$, and M2 macrophages produce much lower levels of TNF- $\alpha$, compared to M1 macrophages. Therefore, we isolated TAM from mice and evaluated the expression of these cytokines by RT-qPCR. TAM from tumors treated with EGCG showed significant decrease of IL- 6 and TGF- $\beta$ level, and higher expression of TNF- $\alpha$ compared with those TAMs from control group (Figure 1E). NF- $k B$ activation in macrophages within tumor microenvironment has been known to differentiate TAM to pro-tumoral and immunosuppressive M2 phenotype during the early stage of tumor development [30]. Because IKK $\alpha$ has a central role for mediating canonical NF- $\mathrm{kB}$ activation pathway, we checked the level of IKK $\alpha$ in TAM from mice by RT-qPCR and found that IKK $\alpha$ mRNA level was significantly decreased in TAM of EGCG-treated group compared with control group (Figure 1F).

Altogether these data suggested that EGCG treatment suppresses in vivo TAM infiltration and inhibits polarization of TAM into tumor-promoting M2 macrophages, which was involved by decreased production of chemo-attractants, CSF-1 and CCL-2, from tumor cells, and down-regulation of M2 macrophage-associated cytokines, IL- 6 and TGF- $\beta$ and relative up-regulation of M1 macrophage-associated cytokine, TNF- $\alpha$, in TMA from EGCG-treated tumor. Inhibition of NF- $\mathrm{kB}$ activity of TAM by EGCG treatment might be implicated in these processes. Importantly, our result suggested that EGCG might exert anti-tumor activity by preventing 


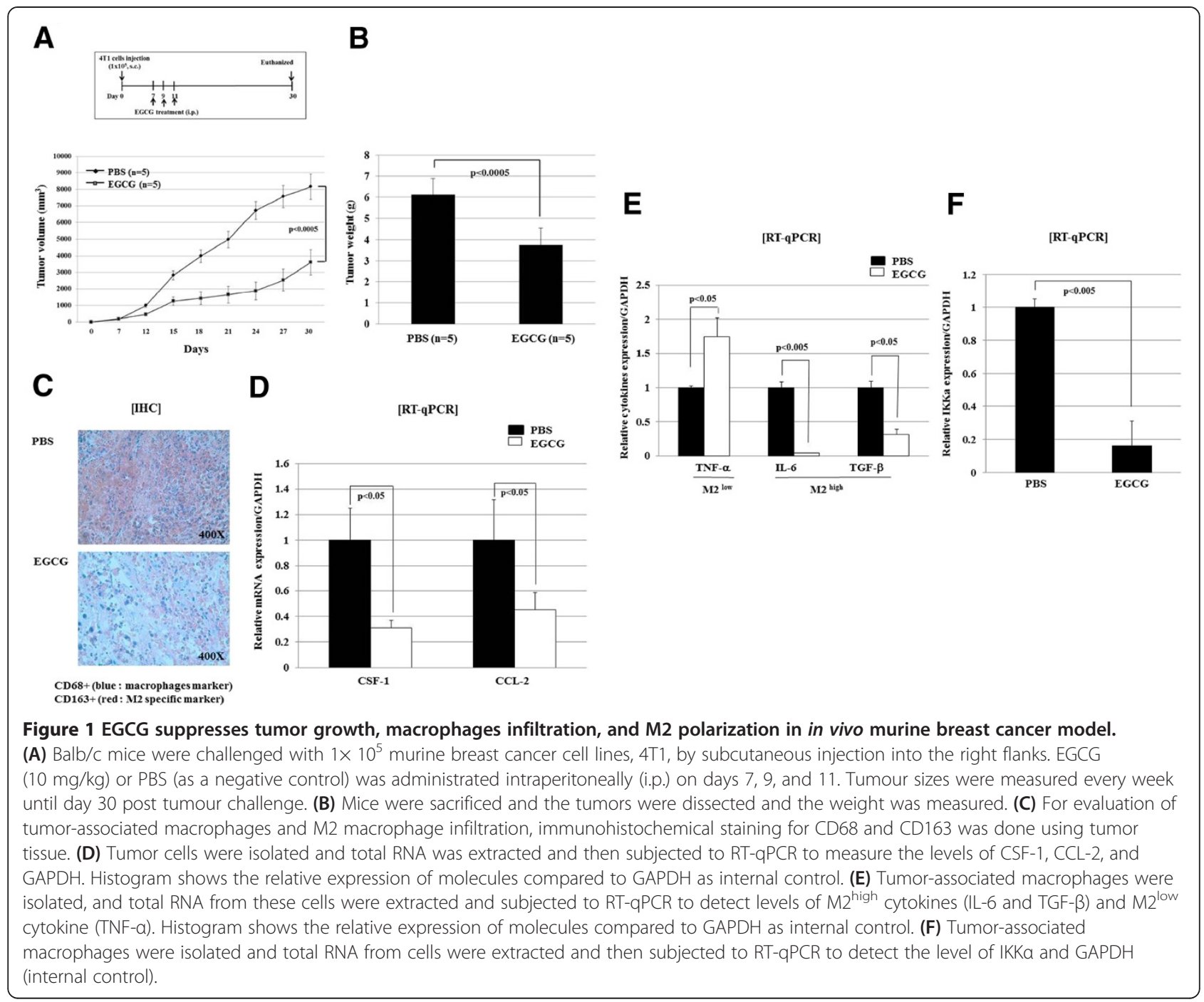

TAM from achieving pro-tumoral properties in tumor microenvironment.

\section{Exosomes derived from EGCG-treated tumor cells leads to decrease of CSF-1 and CCL-2 expression in a paracrine manner, and suppresses M2 polarization of TAM in ex vivo study}

Tumor cells release chemo-attractants to recruit and activate macrophages in a paracrine loop between tumors and macrophages, and this release promotes breast cancer invasion [31]. Exosomes could play an important role of communication between tumor cells and macrophages. In order to determine whether the effect of EGCG observed in the in vivo mouse model could be mediated by tumor exosome, we carried out the ex vivo experiments using the exosomes extracted from 4T1 cell lines. As shown in Figure 2A, treatment of tumor cells with EGCG resulted in a significant decrease of both CSF-1 and CCL-2 expression. In addition, ex vivo incubation of TAM with exosomes from EGCG-treated 4T1 cells skewed polarized these macrophages away from the tumor-promoting M2to a tumor-inhibiting M1-like phenotype, as evidenced by down-regulated the expression of IL- 6 and TGF- $\beta$, but up-regulated TNF- $\alpha$ expression (Figure 2B). We confirmed the protein levels of IL-6, TGF- $\beta$, and TNF- $\beta$ and obtained results same as in RT-qPCR (Figure $2 \mathrm{C}$ ). These data indicated that exosomes derived from EGCG-treated tumor cells suppress TAM infiltration and inhibit TAM differentiation into M2 macrophages in murine breast cancer model.

EGCG up-regulates cellular and exosomal miR-16 levels in murine breast cancer cells, 4T1

To investigate the mechanism by which EGCG treatment had led to decreased TAM infiltration and inhibition of M2 polarization via exosomes, we focused on the effect of EGCG on the regulation of tumor exosomal miRNA. We hypothesized that EGCG might change the 


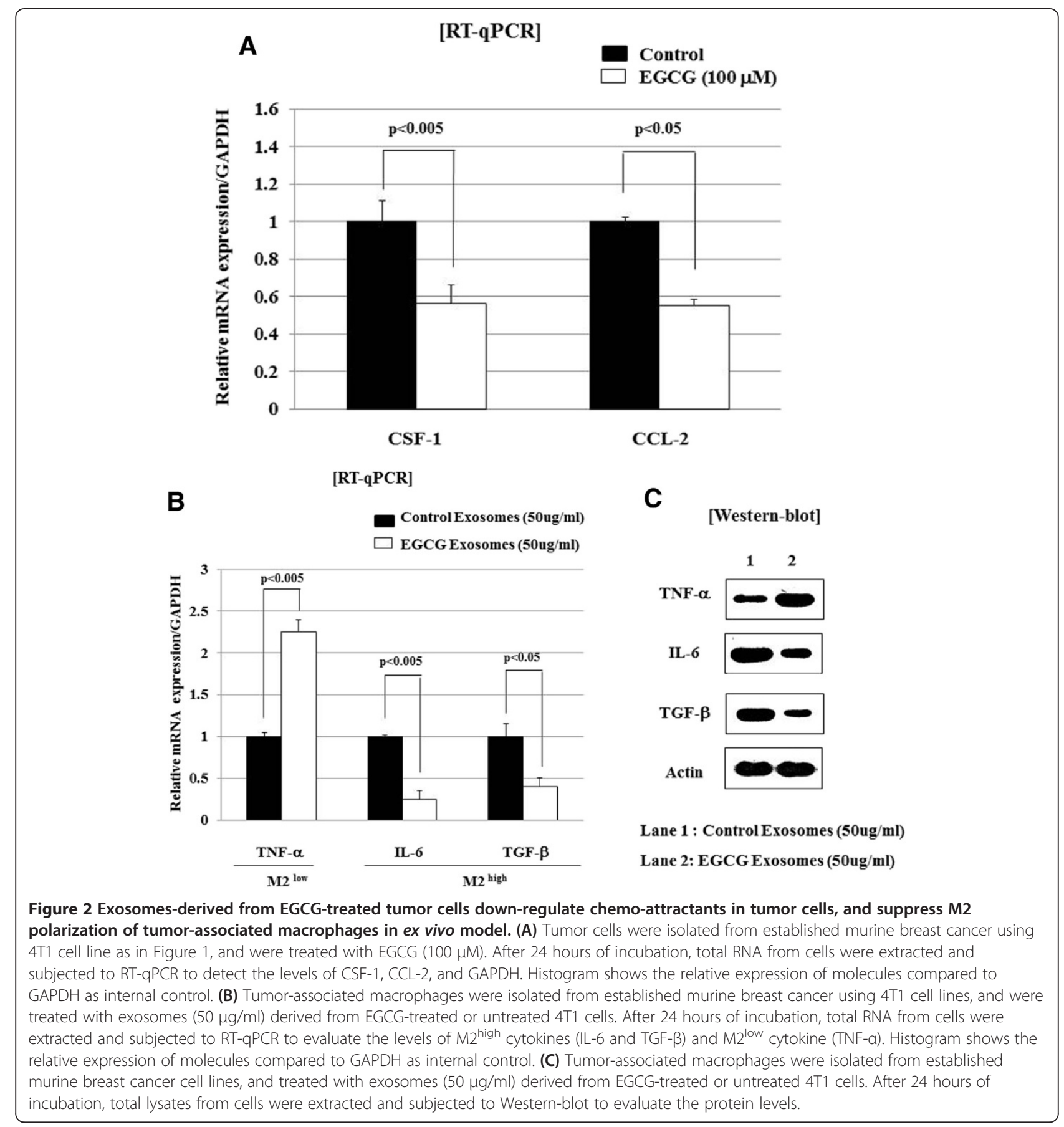

miRNA expression in tumor cells and subsequently in exosomal compartment, and that the exosomal miRNA to TAM would influence on the recruitment and differentiation of TAM. To address this issue, we first screened the miRNAs whose expressions are modulated in $4 \mathrm{~T} 1$ cells by miRNA microarray analysis using both total cellular miRNA and exosomal miRNA after treatment with $100 \mu \mathrm{M}$ of EGCG for $24 \mathrm{~h}$. In brief, a set of miRNAs including let-7, miR-16, miR-18b, miR-20a,
miR-25, miR-92, miR-93, miR-221, and miR-320 were up-regulated, and dozens of miRNAs including miR-10a, miR-18a, miR-19a, miR-26b, miR-29b, miR-34b, miR-98, miR-129, miR-181d were down-regulated in both total cellular and exosomal fraction by EGCG treatment (data not shown). Of these, miRNAs up-regulated by EGCG were suitable for further in vitro study because it is more likely that over-expressed miRNA could be stored within exosome and then transferred to TAM. Specifically, the 
miR-16 was selected because it was elevated by EGCG treatment and has been known to be associated with immune cell function.

To validate the microarray data, $4 \mathrm{~T} 1$ cells were incubated with EGCG, and the total RNA was extracted from cells and secretory exosomes. This was then analyzed by RT-qPCR. Significant up-regulation of miR-16 in both EGCG-treated cells and exosomes was observed with a 1.45 and 2.54 folds change compared with the levels of controls ( $\mathrm{p}<0.05$ and $\mathrm{p}<0.005$, respectively) (Figure 3).

\section{Up-regulation of exosomal miR-16 by EGCG treatment down-regulates IKKa and subsequently induces IKB accumulation in TAM, and inhibits M2 polarization}

MiR-15a and miR-16 have been known to act as a negative regulator of NF- $\mathrm{KB}$ activity by regulating IKK $\alpha$ expression, which contributes to the ability of miR-15 and niR-16 as a tumor suppressor. NF- $\mathrm{kB}$ activation is also important for monocyte differentiation into macrophage and TAM. In fact, a study has reported that during monocyte-macrophage differentiation, expressions of miR-15a and miR-16 were decreased with higher expression of the IKK $\alpha[30]$.

As already known, we identified the possible target sequence in the 3 '-UTR of IKK $\alpha$ for miR-16. Therefore, we tested whether exosomes derived from EGCG- treated 4T1 cells could suppress IKK $\alpha$ expression and, if so, whether that process occurs through exosomal miR-16. As shown in Figure 4A, the treatment of TAM with exosomes from EGCG-treated 4T1 cells has resulted in decreased IKK $\alpha$ protein expression and concomitant accumulation of the I- $\mathrm{KB}$ in TAM. Moreover, when TAM was incubated with exosomes from EGCGtreated and miR-16 inhibitor-transfected 4T1 cells, IKK $\alpha$ levels had recovered to the that of the control (4T1 exosome treated TAM) (Figure 4B). MiR-16 expression was not elevated in exosomes from EGCG-treated and miR-16 inhibitor-transfected 4T1 cells (data not shown), unlike exosomes from EGCG-treated 4T1 cells where miR-16 was up-regulated by EGCG (Figure 3).

To test whether exosome from EGCG-treated 4T1 cells could inhibit M2 polarization of TAM through exosomal miR-16, we treated TAM with exosomes either from EGCG-treated 4T1 cells or from from EGCGtreated and miR-16 inhibitor-transfected 4T1 cells, and measured the level of cytokines including IL-6, TGF- $\beta$, and TNF- $\alpha$. Consistent with the in vivo data (Figure 1E), incubation of TAM with exosomes from EGCG-treated 4T1 cells had led to the suppression of M2-associated cytokines, IL- 6 and TGF- $\beta$, and elevation of the M1related cytokine, TNF- $\alpha$ (Figure $4 \mathrm{C}$ ). More importantly, this alteration of cytokines was restored when TAM was incubated with exosomes from EGCG-treated and miR-

\section{[RT-qPCR]}

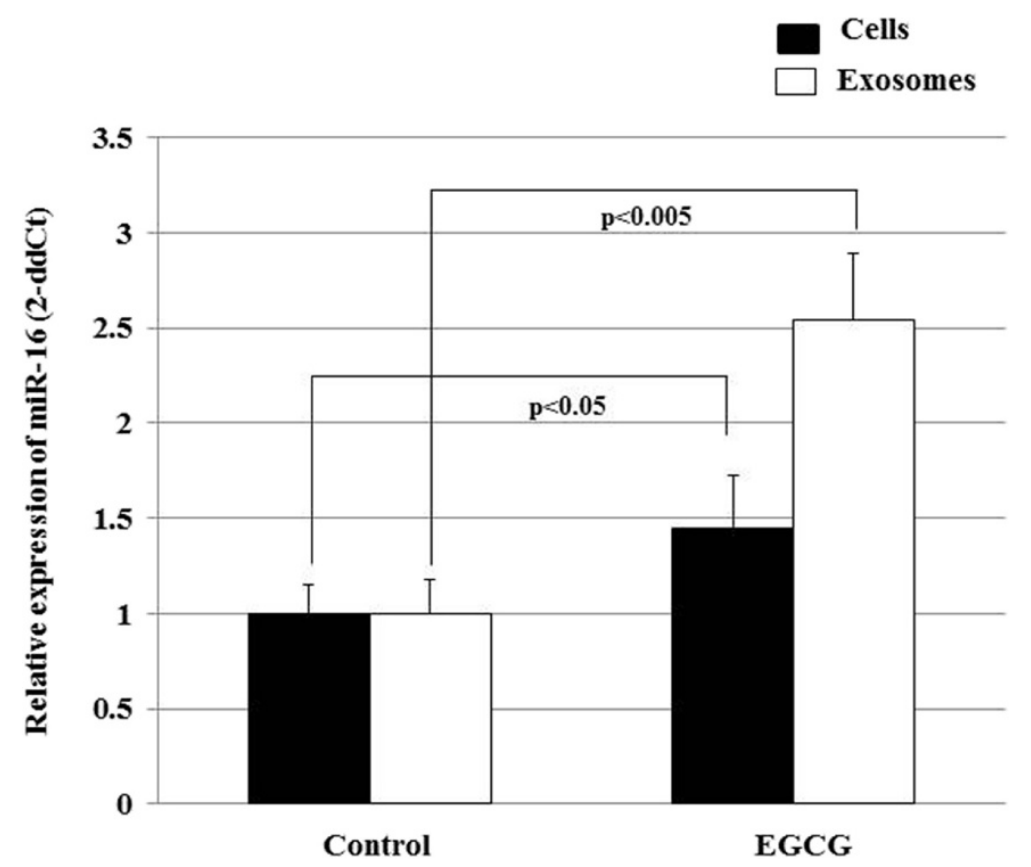

Figure 3 EGCG increases cellular and exosomal miR-16 expression in murine breast cancer cell line, 4T1. 4T1 cells were treated with and without EGCG $(100 \mu \mathrm{M})$ for 48 hours. Then, total RNA from cells or secretory exosomes was extracted and subjected to RT-qPCR to assess the miR-16 level. Histogram shows the relative expression of miR-16 compared to U6 as an internal control. 


\section{[Western-blot]}

A

\section{[Western-blot]}

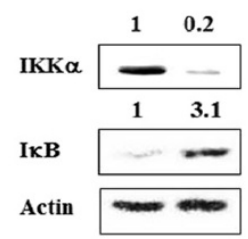

Lane 1 : 4T1 exosomes

Lane 2 : EGCG treated 4T1 exosomes
B

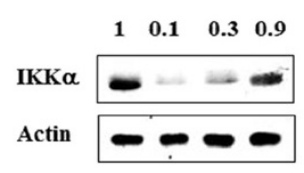

Lane 1 : 4T1 exosomes

Lane 2: EGCG treated 4T1 exosomes

Lane 3 : EGCG treated and scramble miR inhibitor transfected 4T1 exosomes

Lane 4 : EGCG treated and miR-16 inhibitor transfected 4T1 exosomes
C

\section{[RT-qPCR]}

4T1 exosomes

EGCG treated 4T1 exosomes

Scramble miR inhibitor transfected $4 \mathrm{~T} 1$ exosomes

EGCG treated and miR-16 inhibitor transfected 4T1 exosomes

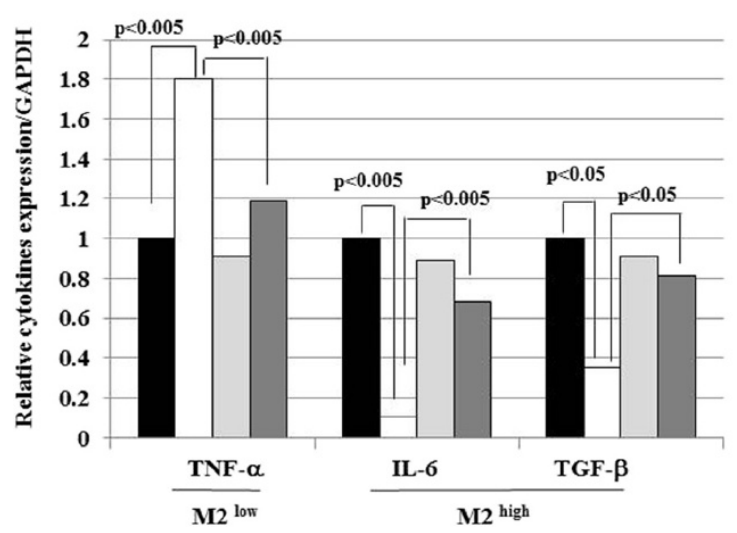

[RT-qPCR]

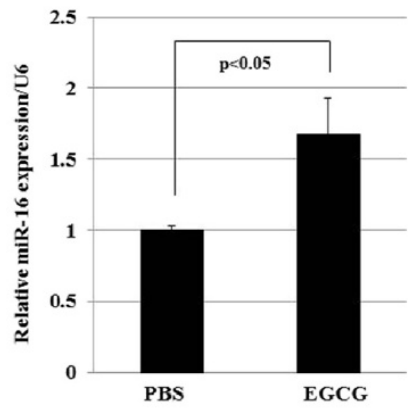

Figure 4 Exosomes derived from EGCG-treated tumor cells suppresses NF-KB pathway and inhibits M2 polarization of tumorassociated macrophages, which is dependent on up-regulated miR-16 in EGCG-treated tumor exosomes. (A) Tumor-associated macrophages were isolated from established murine breast cancer 4T1 cell lines, and treated with exosomes extracted from EGCG (100 $\mu M$ )treated and untreated $4 \mathrm{~T} 1$ cells for 24 hours. Whole cell lysates were prepared and submitted to Western blot using anti-IKKa, anti-I-KB or anti-actin antibodies. (B) 4T1 cells were incubated with EGCG $(100 \mu \mathrm{M})$ and simultaneously (24 hours) transfected with scramble or miR-16 inhibitor for 24 hours. Exosomes were then extracted from each tumor cells. Tumor-associated macrophages isolated from established murine breast cancer were treated with each exosome as indicated at the concentration of $50 \mu \mathrm{g} / \mathrm{ml}$ for 24 hours, and whole cell lysates were prepared and submitted to Western blot using anti-IKKa or anti-actin antibodies. The numbers in Figure $4 \mathrm{~A}$ and $4 \mathrm{~B}$ report the quantification of bands by imaging analysis with KODAK Molecular Imaging Software (MI). (C) 4T1 cells were incubated with EGCG (100 $\mu \mathrm{M})$ and were simultaneously (24 hours) transfected with scramble or miR-16 inhibitor for 24 hours. Exosomes were then extracted from each tumor cells. Tumor-associated macrophages isolated from established murine breast cancer were treated with each exosome at the concentration $50 \mathrm{mg} / \mathrm{ml}$ for 24 hours, and total cellular RNA was extracted and submitted to RT-qPCR analysis for M2 ${ }^{\text {high }}$ cytokines (IL-6 and TGF- $\beta$ ) and M2 ${ }^{\text {low }}$ cytokine (TNF-a). Histogram shows the relative expression of molecules compared to GAPDH as internal control. (D) Tumor cells were isolated and total RNA from cells were extracted and subjected to RT-qPCR to measure the miR-16 level. Histogram shows the relative expression of miR-16 compared to U6 as an internal control.

16 inhibitor-transfected 4T1 cells (Figure 4C). We verified that the levels of miR-16 are reduced by transfection of miR-16 inhibitor (Additional file 1). Finally, we observed a 1.68-fold increase of miR-16 expression in tumor cells from mice treated with EGCG compared to control (Figure 4D). To investigate modulation of 
macrophage by miR-16, mouse macrophage cell line, RAW264.7, cells was transfected with scramble or miR-16 mimics and stimulated with $5 \mu \mathrm{g} / \mathrm{ml}$ LPS. We found that miR-16 mimics significantly suppressed LPS-induced IL-1 $\beta$ and IL-6 production in these RAW264.7 cells (Additional file 2).

Together, the results suggest that EGCG treatment leads to up-regulation of miR-16, which might be transferred by exosomes to TAMs, and contributes to the suppression of NF- $\mathrm{kB}$ activity by down-regulating IKK $\alpha$ and subsequently accumulating Ік-B, and inhibition of TAM infiltration and M2 polarization. These molecular pathways might represent a new mechanism by which EGCG exerts anti-tumor effect through manipulating TAM and tumor microenvironment, as illustrated in Figure 5.

\section{Discussion}

Exosomes are vesicles with a diameter of $50-100 \mathrm{~nm}$ secreted from intracellular endosomes. These vesicles are unrelated to the RNA exosome, which is an RNAprocessing intercellular complex. Membrane vesicles, which are also referred to as microvesicles or microparticles, have a diameter of $100-1,000 \mathrm{~nm}$ and are presumed to be formed by budding or shedding from plasma membrane [32]. Studies of the functional contributions of these vesicles to intercellular communication have focused on understanding the role of their membranous and cytoplasmic protein content. A role in the modulation of immune function has also emerged because the cellular microenvironment is a critical determinant of tumor progression and development [33].

There is much interest in intercellular communication between tumor cells and immune cells in the tumor microenvironment. Cells use a variety of approaches to communicate with each other, such as direct membraneto-membrane contact or by the release of soluble mediators. Among these various means of information transfer, exosomes represent a unique way to mediate cell-to-cell communication [34-38]. Exosomes contain membrane proteins similar to those of the donor cell and contain protein and RNA derived from the donor cell cytoplasm [39]. These vesicles can be taken up and transfer the intravesicular content into the host intracellular environment, which subsequently modulates cellular activities of the recipient cells. Thus, exosomes provides cells with the ability to signal and transfer the intracellular molecular content within the local microenvironment as well as to distant locations [40].

Recently, it has been found that exosome is an important physiologic vehicle carrying and delivering miRNA. When introduced into a new cytoplasmic space, mature miRNAs associate with the RNA-induced silencing complex to effect gene expression [41]. The selective enrichment of these miRNAs in cellular exosomes, the consistency in expression between different isolations, and the cell-type specificity indicate the presence of a mechanism for their active elaboration within these particles. This may arise from selective transport into a membrane-bound exosome or from sequestration of proteins that are selectively enriched within the exosome. Alternatively, the possibility exists that these miRNAs are rapidly degraded within the cytoplasm, but are protected from degradation when they are sequestered in vesicles before their elaboration from cells as cellular exosomes. Because the tumor cells studied vary in cellular behavior, it is not unexpected that some differences in miRNA content were noted between various cell types [42]. These observations regarding cell-type specificity of miRNA content are similar to those made

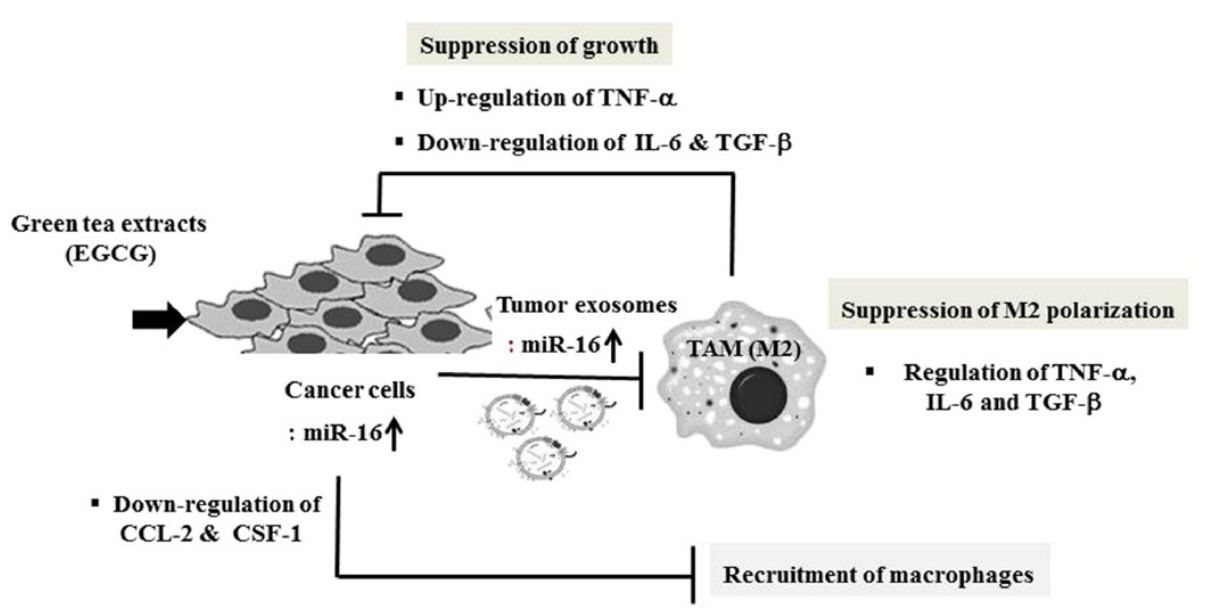

Figure 5 Proposed mechanisms of EGCG to inhibit infiltration of tumor-associated macrophages and M2 polarization in tumor microenvironment. 
with respect to protein content of exosomes. They emphasize the need to study and interpret data on individual cell-type basis.

In the present study, a selective set of miRNA was present within EGCG-treated exosomes which was markedly different from that from untreated breast cancer cells. Among the miRNAs by modulated by EGCG, we focused on miR-16 because it had been identified as one of the down-regulated miRNAs in murine and human breast cancer cells. Additionally, targets of miR-16 include many genes related to the control of cell-cycle progression, such as cyclin D1, cyclin E, and the anti-apoptotic protein, $\mathrm{Bcl}-2$ [43-45]. The restoration of miR-16 in prostate cancer cells results in growth arrest, apoptosis and in marked regression of prostate tumor xenografts [45]. A therapeutic strategy is underway that involves the delivery of synthetic miR-16 into advanced prostate tumors [46]. Overexpression of miR-16 was shown to suppress the selfrenewal and growth of mouse breast tumor stem cells and to sensitize MCF-7 human breast cancer cells to the chemotherapeutic drug doxorubicin [47]. In addition to the role of tumor suppressor, miR-16 plays a role in macrophages. For example, IKK $\alpha$ mRNA is a target for miR-15 and miR-16. During monocyte-to-macrophage differentiation, a substantial decrease in these miRNAs allows for a considerable increase in IKK $\alpha$ protein and for the subsequent activation of NF-kB pathway [29]. In this study, we have revealed for the first time that EGCG modulates the miRNA profile within tumor exosomes and upregulates miR-16, which was responsible for EGCG-treated exosome down-regulating IKK $\alpha$ and inhibiting M2 polarization of TAM. Considering that miR-16 can function as a tumor suppressor, it is possible that up-regulated exosomal miR-16 might also have had an effect on the survival and proliferation of tumor cells in our in vivo experiment.

Macrophage infiltration was decreased by EGCG treatment in the mouse tumor model. To claim that these results are originated from the EGCG-mediated inhibition of macrophage recruitment and M2 polarization rather than the effect of EGCG on macrophage proliferation, we treated TAM isolated from mouse with exosome from EGCG-treated $4 \mathrm{~T} 1$ cell lines. In these ex vivo experiments, we did not observe any decrease in macrophage numbers. However, the mobility of macrophage (data not shown) and the change of phenotype toward non-M2-like macrophages were found to be decreased.

It has been known that NF- $\mathrm{KB}$ activation is important for macrophage-to-M2 macrophage differentiation and contributes to tumor progression. Therefore NF- $\mathrm{kB}$ in TAM is considered as a novel therapeutic target for cancer control. However, several studies have recently suggested that NF- $\mathrm{kB}$ in TAM could have a more complicated and multifaceted role during tumor initiation and progression [48]. For example, in an established murine fibrosarcoma model, the maintenance of M2phenotype was associated with defective NF- $\mathrm{kB}$ activation. This paradoxical role of NF- $\mathrm{kB}$ in TAM requires further investigation, and should be addressed in a tumor model-specific manner. The ex vivo experiments, using macrophages from established murine breast tumor instead of blood monocytes, would reflect the effect of EGCG on TAM during tumor progression rather than tumor initiation. However for the in vivo experiment, EGCG was administrated at the early period after tumor transplantation. We observed the down-regulation of NF-kB pathway in terms of $\mathrm{IKK} \alpha$ and $\mathrm{I}-\kappa \mathrm{B}$ expression in TAM by EGCG or EGCG-treated exosomes in vivo and ex vivo, which we think would be the molecular mechanism underling the EGCG-mediated hindrance of infiltration and differentiation of macrophages into tumor-promoting M2 macrophages, although direct evidences are lacking.

EGCG has been reported to have anticancer biological activity as well. The main mechanism of this activity involves the inhibition of cell proliferation and induction of apoptosis [34,35]. EGCG can inhibit the cellular proliferation in skin cancer, lung cancer, oral cancer, gastric cancer, intestinal cancer, colon cancer, hepatocellular carcinoma, pancreatic cancer, rectal cancer, prostate cancer and breast cancer, suggesting that EGCG could be utilized as a potential anti-cancer drug $[16,36,37]$. However, regulation of exosomal miRNAs by EGCG in tumor cells has not previously been studied. We sought to evaluate the ability of breast cancer cells to release vesicles capable of modulating immune response and to investigate modulation of these vesicles by treatment with EGCG. In the present study, we showed that EGCG can modulate the miRNA contained within exosomes and suppresses immune response, and particularly tumor-associated macrophages. The significance of the experiments in this study is that the mechanism by which EGCG mediates communication between the tumor cells and immune cells has been revealed for the first time. Whether this scenario is applicable to other tumors remains to be elucidated.

\section{Conclusions}

In this study, we demonstrated that EGCG can suppress tumor growth through the inhibition of TAM infiltration and M2 polarization, using in vivo and ex vivo murine breast cancer model. Moreover, we revealed that EGCG modulates miRNAs, particularly upregulates miR-16, which is transferred to adjacent tumor cells and TAM via tumor-derived exosomes and which has an influence on macrophages in tumor microenvironment. 


\section{Additional files}

Additional file 1: 4T1 cells were incubated with EGCG (100 $\mu \mathrm{M})$ and simultaneously ( 24 hours) transfected with scramble or miR-16 inhibitor for $\mathbf{2 4}$ hours. Exosomes were then extracted from each tumor cells, and total RNA from these cells were extracted and subjected to RT-qPCR to measure the miR-16 level. Histogram shows the relative expression of miR-16 compared to U6 as an internal control.

Additional file 2: RAW264.7 cells were stimulated with $5 \mu \mathrm{g} / \mathrm{ml}$ LPS in the transfection scramble or miR-16 mimics, and total cellular RNA was extracted and submitted to RT-qPCR analysis for IL-1 $\beta$ and IL-6. Histogram shows the relative expression of molecules compared to GAPDH as internal control.

\section{Abbreviations}

EGCG: Epigallocatechin gallate; TAM: Tumor-associated macrophages.

\section{Competing interests}

The authors declare that they have no other competing interests.

\section{Authors' contributions}

$J-Y J$ and J-KL have performed the majority of the experiments and were responsible for generating the results, the data analysis, and preparing the manuscript. Y-KJ was responsible for data analysis and for writing the paper. C-WK contributed to the design of the project, data analysis, and writing the manuscript. All authors have reviewed and agreed to the content of the final, submitted version of the manuscript.

\section{Acknowledgements}

This work was supported by the Global Core Research Center (GCRC) grant (No. 2012-0001190) from the National Research Foundation (NRF), Ministry of Education, Science and Technology (MEST) (Republic of Korea).

Received: 8 November 2012 Accepted: 9 September 2013 Published: 17 September 2013

\section{References}

1. Gezgen G, Roach EC, Kizilarslanoglu MC, Petekkaya I, Altundag K: Metabolic syndrome and breast cancer: an overview. J Buon 2012, 17:223-229.

2. Kelsey JL, Gammon MD: The epidemiology of breast cancer. CA Cancer J Clin 1991, 41:146-165.

3. Key TJ, Schatzkin A, Willett WC, Allen NE, Spencer EA, Travis RC: Diet, nutrition and the prevention of cancer. Public Health Nutr 2004, 7:187-200.

4. Yang CS, Sang S, Lambert JD, Hou Z, Ju J, Lu G: Possible mechanisms of the cancer-preventive activities of green tea. Mol Nutr Food Res 2006, 50:170-175.

5. Mukhtar H, Ahmad N: Tea polyphenols: prevention of cancer and optimizing health. Am J Clin Nutr 2000, 71:1698S-1702S.

6. Lambert JD, Hong J, Yang GY, Liao J, Yang CS: Inhibition of carcinogenesis by polyphenols: evidence from laboratory investigations. Am J Clin Nutr 2005, 81:284S-291S.

7. Stuart EC, Scandlyn MJ, Rosengren RJ: Role of epigallocatechin gallate (EGCG) in the treatment of breast and prostate cancer. Life Sci 2006, 2006(79):2329-2336.

8. Zhang G, Miura Y, Yagasaki K: Effects of green, oolong and black teas and related components on the proliferation and invasion of hepatoma cells in culture. Cytotechnology 1999, 31:37-44.

9. Lu G, Liao J, Yang G, Reuhl KR, Hao X, Yang CS: Inhibition of adenoma progression to adenocarcinoma in a 4-(methylnitrosamino)-1-(3-pyridyl)1-butanone-induced lung tumorigenesis model in $\mathrm{A} / \mathrm{J}$ mice by tea polyphenols and caffeine. Cancer Res 2006, 66:11494-11501.

10. Lu YP, Lou YR, Xie JG, et al: Topical applications of caffeine or (-)-epigallocatechin gallate (EGCG) inhibit carcinogenesis and selectively increase apoptosis in UVB-induced skin tumors in mice. Proc Natl Acad Sci U S A 2002, 99:12455-12460.

11. Thangapazham RL, Singh AK, Sharma A, Warren J, Gaddipati JP, Maheshwari RK: Green tea polyphenols and its constituent epigallocatechin gallate inhibits proliferation of human breast cancer cells in vitro and in vivo. Cancer Lett 2007, 245:232-241.
12. Adhami VM, Siddiqui IA, Ahmad N, Gupta S, Mukhtar H: Oral consumption of green tea polyphenols inhibits insulin-like growth factor-l-induced signaling in an autochthonous mouse model of prostate cancer. Cancer Res 2004, 64:8715-8722.

13. Wang $Z Y$, Huang MT, Ho CT, et al: Inhibitory effect of green tea on the growth of established skin papillomas in mice. Cancer Res 1999, 52:6657-6665.

14. Heijnen HF, Schiel AE, Fijnheer R, et al: Activated platelets release two types of membrane vesicles: microvesicles by surface shedding and exosomes derived from exocytosis of multivesicular bodies and alphagranules. Blood 1999, 94:3791-3799.

15. Rozmyslowicz T, Majka M, Kijowski J, et al: Platelet- and megakaryocytederived microparticles transfer CXCR4 receptor to CXCR4null cells and make them susceptible to infection by X4-HIV. AIDS 2003, $17: 33-42$.

16. Majka M, Janowska-Wieczorek A, Ratajczak J, et al: Numerous growth factors, cytokines, and chemokines are secreted by human CD34(+) cells, myeloblasts, erythroblasts, and megakaryoblasts and regulate normal hematopoiesis in an autocrine/paracrine manner. Blood 2001 97:3075-3085.

17. Kim HK, Song KS, Park YS, Kang YH, Lee YJ, Lee KR, Kim HK, Ryu KW, Bae JM, Kim S: Elevated levels of circulating platelet microparticles, VEGF, IL-6 and RANTES in patients with gastric cancer: possible role of a metastasis predictor. Eur J Cancer 2003, 39:184-191.

18. lero M, Valenti R, Huber $V$, Filipazzi P, Parmiani G, Fais S, Rivoltini L: Tumour-released exosomes and their implications in cancer immunity. Cell Death Differ 2008, 15:80-88.

19. Safaei R, Larson BJ, Cheng TC, Gibson MA, Otani S, Naerdemann W, Howell SB: Abnormal lysosomal trafficking and enhanced exosomal export of cisplatin in drug-resistant human ovarian carcinoma cells. Mol Cancer Ther 2005, 4:1595-1604.

20. Millimaggi D, Mari M, D'Ascenzo S, Carosa E, Jannini EA, Zucker S, Carta G, Pavan A, Dolo V: Tumor vesicle-associated CD147 modulates the angiogenic capability of endothelial cells. Neoplasia 2007, 9:349-357.

21. Camussi G, Deregibus MC, Bruno S, Grange C, Fonsato V, Tetta C: Exosome/ microvesicle-mediated epigenetic reprogramming of cells. Am J Cancer Res 2011, 1:98-110.

22. Pollard JW: Macrophages define the invasive microenvironment in breast cancer. J Leukoc Biol 2008, 84:623-630.

23. Bingle $L$, Brown NJ, Lewis CE: The role of tumor-associated macrophages in tumor progression: implications for new anticancer therapies. J Pathol 2002, 196:254-265.

24. Leek RD, Harris AL: Tumor-associated macrophages in breast cancer. J Mammary Gland Biol Neoplasia 2002, 7:177-189.

25. Antonio S, Tiziana S, Alberto M, Paola A: Tumour-associated macrophages are a distinct $M 2$ polarised population promoting tumour progression: Potential targets of anti-cancer therapy. Eur J Cancer 2006, 42:717-727.

26. Saccani A, Schioppa T, Porta C, Biswas SK, Nebuloni M, Vago L, Bottazzi B, Colombo MP, Mantovani A, Sica A: p50 nuclear factor-kB overexpression in tumor-associated macrophages inhibits $\mathrm{M} 1$ inflammatory responses and antitumor resistance. Cancer Res 2006, 66:11432-11440.

27. Mantovani A, Marchesi F, Porta C, Sica A, Allavena P: Inflammation and cancer: breast cancer as a prototype. Breast 2007, 16:S27-S33.

28. Paik S, Shak S, Tang G, et al: A multigene assay to predict recurrence of tamoxifen-treated, node-negative breast cancer. N Engl J Med 2004, 351:2817-2826.

29. Li T, Morgan MJ, Choksi S, Zhang Y, Kim YS, Liu ZG: MicroRNAs modulate the noncanonical NF-KB pathway by regulating IKKa expression during macrophage differentiation. Nat Immunol 2010, 11:799-805.

30. Hagemann T, Lawrence T, McNeish I, Charles KA, Kulbe H, Thompson RG, Robinson SC, Balkwill FR: Re-educating tumor-associated macrophages by targeting NF-kappaB. J Exp Med 2008, 205:1261-1268.

31. Shih JY, Jeremy AY, Chen JW, Yang PC: Tumor-associated macrophage: its role in cancer invasion and metastasis. J Cancer Mol 2006, 2:101-106.

32. Bobrie A, Colombo M, Krumeich S, Raposo G, Théry C: Diverse subpopulations of vesicles secreted by different intracellular mechanisms are present in exosome preparations obtained by differential ultracentrifugation. J Extracellular Vesicles 2012, 1:18397.

33. Chew V, Toh HC, Abastado JP: Immune microenvironment in tumor progression: characteristics and challenges for therapy. J Oncol 2012, 2012:1-10. 
34. Théry C: Exosomes: secreted vesicles and intercellular communications. F1000 Biology Reports 2011, 3:15.

35. Camussi G, Deregibus MC, Bruno S, Cantaluppi V, Biancone L: Exosomes/ microvesicles as a mechanism of cell-to-cell communication. Kidney Int 2010, 78:838-848.

36. Rustom A, Saffrich R, Markovic I, et al: Nanotubular highways for intercellular organelle transport. Science 2004, 303:1007-1010.

37. Sherer NM, Mothes W: Cytonemes and tunnelling nanotubules in cell-cell communication and viral pathogenesis. Trends Cell Biol 2008, 18:414-420.

38. Ratajczak J, Wysoczynski M, Hayek F, et al: Membrane-derived microvesicles: important and underappreciated mediators of cell-to-cell communication. Leukemia 2006, 20:1487-1495.

39. Hutagalung AH, Novick PJ: Role of Rab GTPases in membrane traffic and cell physiology. Physiol Rev 2011, 91:119-149.

40. Skog J, Wurdinger T, van Rijn S, Meijer DH, Gainche L, Sena-Esteves M, et al: Glioblastoma microvesicles transport RNA and proteins that promote tumour growth and provide diagnostic biomarkers. Nat Cell Biol 2008, 10:1470-1476.

41. Pillai RS: MicroRNA function: multiple mechanisms for a tiny RNA? RNA 2005, 11:1753-1761.

42. Pigati L, Yaddanapudi SC, lyengar R, Kim DJ, Hearn SA, Danforth D, Hastings ML, Duelli DM: Selective release of MicroRNA species from normal and malignant mammary epithelial cells. PLoS One 2010, 5:e13515.

43. Liu Q, Fu H, Sun F, Zhang H, Tie Y, Zhu J, Xing R, Sun Z, Zheng X: miR-16 family induces cell cycle arrest by regulating multiple cell cycle genes. Nucleic Acids Res 2008, 36:5391-5404.

44. Ofir M, Hacohen D, Ginsberg D: miR-15 and miR-16 are direct transcriptional targets of E2F1 that limit E2F-induced proliferation by targeting cyclin E. Mol Cancer Res 2011, 9:440-447.

45. Bonci D, Coppola V, Musumeci M, Addario A, Giuffrida R, Memeo L, D'Urso L, Pagliuca A, Biffoni M, Labbaye C, Bartucci M, Muto G, Peschle C, De Maria R: The miR-15a-miR-16-1 cluster controls prostate cancer by targeting multiple oncogenic activities. Nat Med 2008, 14:1271-1277.

46. Takeshita F, Patrawala L, Osaki M, Takahashi RU, Yamamoto Y, Kosaka N, Kawamata M, Kelnar K, Bader AG, Brown D, Ochiya T: Systemic delivery of synthetic microRNA-16 inhibits the growth of metastatic prostate tumors via downregulation of multiple cell-cycle genes. Mol Ther 2010, 18:181-187.

47. Zhang X, Wan G, Mlotshwa S, Vance V, Berger FG, Chen H, Lu X: Oncogenic Wip1 phosphatase is inhibited by miR-16 in the DNA damage signaling pathway. Cancer Res 2010, 70:7176-7186.

48. Thorsten H, Subhra KB, Toby L, Antonio S, Claire EL: Regulation of macrophage function in tumors: the multifaceted role of NF-KB. Blood 2009, 113:3139-3146

doi:10.1186/1471-2407-13-421

Cite this article as: Jang et al: Exosome derived from epigallocatechin gallate treated breast cancer cells suppresses tumor growth by inhibiting tumor-associated macrophage infiltration and M2 polarization. BMC Cancer 2013 13:421.

\section{Submit your next manuscript to BioMed Central and take full advantage of:}

- Convenient online submission

- Thorough peer review

- No space constraints or color figure charges

- Immediate publication on acceptance

- Inclusion in PubMed, CAS, Scopus and Google Scholar

- Research which is freely available for redistribution

Submit your manuscript at www.biomedcentral.com/submit
( Biomed Central 